\title{
Individuelle Pflegekonzepte je nach Hautalter
}

\begin{abstract}
Das Thema Anti-Aging gewinnt aufgrund der steigenden Lebenserwartung von Frauen immer mehr an Bedeutung. Bei der diesjährigen DDG-Tagung in Dresden diskutierten Expertinnen über spezifische Anti-Aging-Konzepte und die Bedeutung innovativer galenischer Formen und biomimetischer Texturen.
\end{abstract}

Vor jedem Therapiebeginn sollte die individuelle Hautphysiologie bestimmt werden, beschrieb Prof. Martina Kerscher, Hamburg, ihr schrittweises Vorgehen bei der dermatokosmetischen Behandlung. Im zweiten Schritt erfolgt die Differenzierung nach Hautalterungsstufen. Erst danach legt die Dermatologin den konkreten Behandlungsplan fest. Dabei müssen strukturelle Veränderungen wie Elastizitäts- und Volumenverlust, Faltenbildung und funktionelle Veränderungen wie Lipidmangel, Hauttrockenheit und die verminderte Hautschutzbarriere berücksichtigt werden.

Ein täglicher UVA-/UVB-Schutz sollte bei allen Hautalterungsstufen selbstverständlich sein, forderte Kerscher. Zusätzlich werden bereits in der Hautalterungsstufe 1 Zellregulatoren eingesetzt. Der "Goldstandard“ ist laut Kerscher hier Retinol. Als neue verträgliche Retinol-Generation gilt die Kombination des aktiven
Wirkstoffs Retinol mit Adenosin. Adenosin wirkt synergistisch und entspannt Mimikfalten. Dieser Retinol-A-Komplex ist ergänzend zur 3-fach-Dosis Hyaluronsäure in Liftactiv Retinol HA enthalten.

Im weiteren Alterungsverlauf kann eine Verbesserung der Symptome mit Antioxidantien wie Vitamin $\mathrm{C}$ erzielt werden. Gute Ergebnisse werden auch mit dem Wirkstoff Rhamnose (Hauptinhaltsstoff in Liftactiv) erreicht. Das Monosaccharid wirkt über die Signaltransduktion und ist sehr effektiv in der Hautalterungsstufe 2. Es regt die Kollagenbiosynthese an und verbessert die epidermale und dermale Hautdicke.

$\mathrm{Ab}$ etwa 60 Jahren kommt es zu einer hormonell-bedingten Abnahme der Sebumlipid-Produktion. Hier werden biomimetische Texturen mit innovativen Trägermolekülen benötigt, die Ceramide imitieren, Sie verbessern die Verteilung der Lipide im Interzellularraum.
Eine neue Substanzklasse sind die Wachstumsfaktoren-induzierenden Botanicals, zu denen Proteic Gf zählt. Sie führen zu einer signifikanten Verbesserung der Hautelastizität und Neusynthese elastischer Fasern.

Eine andere Substanzgruppe bilden die Pro-Xylane ${ }^{\mathrm{T} m}$. Sie führen in der Epidermis zu einer vermehrten Synthese des Hyaluronsäure-Rezeptors. Zudem stimulieren sie die Kollagenbildung und stärken die Verbindung von Epidermis und Dermis.

Der Wirkstoff-Komplex aus Proteic Gf und Pro-Xylane $^{\mathrm{Tm}}$ ist in Neovadiol Gf, einer Pflege für die reife Haut und in Neovadiol Magistral enthalten. Letzteres enthält zusätzlich pflanzliche Öle, die die Lipiddepots auffüllen. Der Pflegebalsam ist speziell abgestimmt auf die anspruchsvolle, reife Haut in der Hautalterungsstufe 4. $g z$

Nach Information von L'Oréal GmbH, Geschäftsbereich Vichy

\section{Pflege und Schutz für überstrapazierte Hände}

\begin{abstract}
Häufiges Händewaschen, Geschirr spülen, der Umgang mit aggressiven Substanzen oder andere starke Belastungen für die Haut können Trockenheit, Rötungen und sogar Entzündungen verursachen. Werden die Anzeichen von drohenden akuten Hauterkrankungen wie ständige Trockenheit oder etwa Juckreiz nicht ernst genommen, können chronische Erkrankungen und Allergien die Folge sein.
\end{abstract}

Die Hände gelten als Visitenkarte des Menschen und verraten häufig das wahre Alter. Und gerade sie sind besonderen Belastungen ausgesetzt. Um die Haut an den Händen optimal zu pflegen und zu schützen hat der Kosmetikhersteller Pierre Fabre sein Portfolio erweitert: Mit Eau Thermal Avène Cicalfate Hände, $a b$ September exklusiv in Apotheken erhältlich, können die speziellen Bedürf- nisse trockener, übermäßig strapazierter Hände bedient werden. Die cremige und nährende Textur ist transparent und bildet einen wasserfesten Schutzfilm auf der Haut, der langanhaltend vor Trockenheit schützt und die Hautbarriere stärkt. Die feuchtigkeitsspendende Formel mit mikronisiertem Sucralfat regeneriert die Haut, Kupfer - und Zinksulfat wirken adstrinierend und antimykotisch und lassen Dermatosen wie Handekzeme schneller abheilen. Das enthaltene Avène Thermalwasser beruhigt Irritationen, lindert Juckreiz und wirkt entzündungshemmend. Glykole und Emollientien erhöhen die Feuchtigkeitsversorgung.

Nach Informationen von Pierre Fabre 